\title{
How Partner Gender Influences Female Students' Problem Solving in Physics Education
}

\author{
N. Ding, ${ }^{1,2}$ and E. Harskamp ${ }^{1}$
}

\begin{abstract}
Research has shown that female students cannot profit as much as male students can from cooperative learning in physics, especially in mixed-gender dyads. This study has explored the influence of partner gender on female students' learning achievement, interaction and the problem-solving process during cooperative learning. In Shanghai, a total of 50 students (26 females and 24 males), drawn from two classes of a high school, took part in the study. Students were randomly paired, and there were three research groups: mixed-gender dyads (MG), female-female dyads (FF) and male-male dyads (MM). Analysis of students' pre- and post-test performances revealed that female students in the single-gender condition solved physics problems more effectively than did those in the mixed-gender condition, while the same was not the case for male students. We further explored the differences between female and male communication styles, and content among the three research groups. It showed that the females' interaction content and problem-solving processes were more sensitive to partner gender than were those for males. This might explain why mixed-gender cooperation in physics disadvantages females in high schools.
\end{abstract}

KEY WORDS: cooperative learning; gender; interaction; problem solving; physics education.

\section{INTRODUCTION}

In high schools students tend to solve science problems mechanically. They focus on sample problems, search for the correct formula and simply plug numbers into the formula (Sherin, 2001). This kind of symbol manipulation hinders students' acquisition of real problem-solving skills such as creative application and reasoned evaluation of knowledge. Solving problems depends not only on proficiency in recalling knowledge and in using formulas, but also on systematic analysis of information and on critical reflection. Elaboration of

\footnotetext{
${ }^{1}$ Faculty of Behavioral and Social Sciences, University of Groningen, PO Box 12869701 BG, Groningen, The Netherlands ${ }^{2}$ To whom correspondence should be addressed; e-mail: n.ding@ rug.nl
}

knowledge has been evidenced as an important factor in students' problem-solving learning (Sutherland, 2002). Cooperative learning may help students to elaborate on problem information through interpersonal discourse, and it may provoke a higher level of thinking (Johnson and Johnson, 1986). During interaction students are stimulated to put forward and order their thoughts in order to understand the ideas or questions of their peer learner. In this way, elaboration on knowledge seems necessary in order to generate more coherent explanations (Teasley, 1995).

Ever since the 1980s, attempts have been made to apply peer cooperation to problem-solving teaching (Cohen, 1994; Howe et al., 1995; Lehtinen, 2003; Sharan and Shachar, 1988). Mercer (1996) found that students solved problems in a more productive fashion through exploratory talk. According to Schwartz (1995), peer learning provokes more 
abstract representations because of students' different viewpoints. Cooperative learning may lead peers to integrate different perspectives and generate more compounded analyses.

However, simply putting students in a peer group does not mean that they can work together or that cognitive elaboration will take place. In relation to students' interactive processes and learning outcomes, partner gender is an important variable in cooperative learning (Margrett and Marsiske, 2002).

Female and male students have different communication styles (Lakoff, 1973; Lay, 1992; Li, 2002; Webb, 1984, etc.). For example, male students tend to express their opinions directly while female students tend to hedge. Females are more likely to initiate conversation by asking questions, whereas males begin discussions by "presenting explanations."

Research from Hyde et al. (1990) has shown that physics starts to disadvantage female students when they are around 16-year-old. They also found a gender difference favoring male students in high schools, while they found no significant gender difference at the middle-school level. Orenstein (1994) ascribed this to a decrease in confidence and academic risk-taking as girls got older. Males see themselves as the rightful and superior problem-solvers while females think physics is a masculine job. This difference in self-perception and communication style may cause difficulties for female students when working with male partners.

There are some case studies indicating females in single-gender cooperation outperform females in mixed-gender cooperation (Barbieri and Light, 1992; Siann and Macleod, 1986; Siann et al., 1988). The presence of male students seems to make high-school female students reluctant to put forward their ideas and so they become less active in mixed-gender cooperation. Males tend to be dominant while females tend to be submissive.

Experimental studies focusing on female students' cognitive activities during cooperation and how this relates to their problem-solving achievement are sporadic (Hogan and Tudge, 1999). There is no clear empirical evidence on whether female students' interaction style and problem-solving processes are influenced by their partner gender. Therefore, it might be important to pay more attention to the interactive exchanges between female and male students during cooperative learning.

\section{Interaction During Cooperative Learning: Communication Style}

Bales's Interaction Process Analysis model (IPA) (1950, 1999) provides four categories for recording and analyzing the content and intensity of communication. Originally it was designed to investigate leadership styles in group dynamics. Nowadays these categories are used to study interaction styles in cooperative settings (Underwood et al., 1994). The categories included twelve items indicating twelve types of behavior: (a) Social-Emotional Area (positive): showing solidarity, tension release and agreement; (b) Social-Emotional Area (negative): showing disagreement, tension and antagonism; (c) Task Area (questions): asking for orientation, opinion and suggestions; and (d) Task Area (answers): giving orientation, suggestions or opinions. In this study, some modifications were made to make the IPA model fit better into the problem-solving setting.

Understanding students' interaction might extend our knowledge of the gender difference during cooperative learning. For instance, the different communication style of female students brings about problems where their cooperation with males is concerned, especially in subjects that they are not fully confident in like physics problem solving. In this study protocols of students' written interaction were analyzed by means of the modified IPA model. To unravel the gender effects, it also seemed important to gather more evidence of students' communication content both in single-gender and mixed-gender cooperation.

\section{Cognitive Elaboration During Cooperative Learning: Communication Content}

According to Schoenfeld (1992), problem solving is not a strict step-by-step process but involves more flexibility and higher-order thinking. In problem solving Schoenfeld defined five episodes: reading the problem, exploring one's knowledge, planning, implementation and reflecting on the solution. More or less consciously, all students go through these episodes in order to solve problems. During cooperative learning students have to read the problem together and figure out the action plan before they start task-related interaction. To solve the problem in a meaningful way, students need to analyze the problem, for instance by making a schema and attaching appropriate symbols to each important 
parameter in the problem. While planning a solution, students have to map the elements of their knowledge systematically in order to develop a reasoned answer. In physics, mathematical skills are necessary for students to work out the solution plan.

It is assumed that females' lack of self-confidence in physics will exacerbate the latent communication difficulties in mixed-gender dyads. The cognitive exchanges of females with males should mainly be through asking questions. Though we have reasons to believe that mixed-gender dyads run the risk of disadvantaging female students in cognitive elaboration in physics, little is known about the differences of interaction and problem solving between females in mixed-gender and single-gender dyads.

\section{Research Question}

The purpose of the study is to investigate how partner gender influences female student's communication and problem-solving activities in cooperative learning in physics. The research questions are:

1. Does partner gender influence students' learning in problem solving? If so, does it influence female students' learning more than it does that of male students'?

2. Does partner gender influence students' interaction content? If so, how does it influence female and male students' interaction?

3. Does partner gender influence students' problemsolving processes? If so, how does it influence female and male students' problem-solving processes?

\section{METHOD}

\section{Subjects and Design}

Fifty high school students (26 females and 24 males) in Shanghai, along with their physics teacher, participated in the study. Students were selected from two physics classes at grade 11, with a mean age of 16 . This high school ranks among the five best schools in Shanghai. Students there come from various provinces in China and have various family backgrounds.

Students were randomly paired with a peer learner from a different class. There were three pairing combinations on the basis of gender: the mixed-gender condition (MG) included twelve dyads; the female-female condition (FF) included seven dyads and the male-male condition (MM) had six dyads.
The three conditions were exposed to the same number of experimental hours and the same instructional materials. Cross-condition comparison was used to develop insight into students' learning achievements and communication during cooperation. In the following we distinguish four groups: (a) females in $\mathrm{MG}$ conditions, (b) females in FF conditions, (c) males in MG conditions and (d) males in MM conditions.

\section{Procedure}

Two weeks before students sat down together to solve the physics problems, the teacher gave two introductory courses on Newtonian mechanics. Each took $45 \mathrm{~min}$. One week before the experiment all students took a 50 -min pre-test in which they were required to solve five problems individually. Then they were given pre-flight training concerning how to use the communication-log sheets and answer sheets. The experiment consisted of four 45-min-long sessions. In each session, students were asked to solve two new and moderately structured problems. One of the problems is shown in Figure 1.

Twenty-five dyads of students were spread over different classrooms in order to give them ample room for cooperation without disturbing each other. In each classroom there was a teacher or a research assistant overseeing the students at work. In each condition, dyads were not allowed to talk with each other. To communicate with their peer learner students had to write on a piece of blank paper, that is, the communication-log sheet, which was placed between students on the desk. Each dyad was given two pens of different colors, blue and black, to distinguish different students in the dyad. Students were asked to come to mutual agreement on the final answer. Answer sheets were collected by the teacher for grading. The communication-log sheets were handed to the research assistant and not graded. Twenty-five observers were selected from a senior grade. Observers were randomly assigned to each dyad and rotated after each session. The observer's task was to document each dyad's starting and ending time for each problem, and to ensure that each dyad's communication only took place on the communication-log sheets. After the dyad submitted their answer sheet to the teacher, the observer collected their communication-log sheet and verified which color belonged to which student of the dyad, and then gave the dyad a worked-out example. On the last day students took the post-test, solving five problems individually. The only difference among the three conditions was the partner gender. 


Student 1
Name :
Student No. :
Class :
Gender :
A space explorer (1500-kg) rises from the
surface of a certain planet. The pushing force
generated by the motor is constant. When the
explorer is ejected, the motor shuts down
because of some technical problem. As shown
in the picture at right, the speed of the explorer
changes as time goes by. From this picture, can
you tell the maximum height the explorer has
reached and the magnitude of the pushing force
$\mathrm{F}$ generated by the motor?

\begin{tabular}{l|l}
\hline Sample Answer & Scoring (full score=50): \\
\hline
\end{tabular}

1. $0-8 \mathrm{~s}$

$v_{t}=40 \mathrm{~m} / \mathrm{s} \quad v_{0}=0 \mathrm{~m} / \mathrm{s} \quad \mathrm{t}=8 \mathrm{~s} \rightarrow \mathrm{a}=\left(\mathrm{v}_{\mathrm{t}}-\mathrm{v}_{\mathrm{o}}\right) / \mathrm{t}=40 / 8=5 \mathrm{~m} / \mathrm{s}^{2} \quad 5$

\begin{tabular}{ll}
\hline $\mathrm{F}_{\text {resultant }}=\mathrm{ma}=1500^{*} 5=7500 \mathrm{~N}$ & 5 \\
\hline $\mathrm{F}_{\text {resultant }}=\mathrm{F}_{\text {push }}-\mathrm{F}_{\text {gravity }}$ & 5
\end{tabular}

2. $8-24 \mathrm{~s}$

$v_{0}=40 \mathrm{~m} / \mathrm{s} \quad v_{t}=0 \mathrm{~m} / \mathrm{s} \quad t=(24-8)=16 \mathrm{~s} \rightarrow \mathrm{g}=\left(\mathrm{v}_{\mathrm{t}}-\mathrm{v}_{\mathrm{o}}\right) / \mathrm{t}=40 / 16=2.5 \mathrm{~m} / \mathrm{s}^{2} \quad 6$

$\mathrm{F}_{\text {gravity }}=\mathrm{mg}=1500^{*} 2.5=3750 \mathrm{~N}$

$\mathrm{F}_{\text {push }}=\mathrm{F}_{\text {resultant }}+\mathrm{F}_{\text {gravity }} \quad 10$

$=7500+3750 \quad 5$

$=11250 \mathrm{~N}$

$\begin{array}{ll}\mathrm{H}=1 / 2 \mathrm{vt} & 5 \\ =1 / 2 * 40^{*} 24 & 5\end{array}$

$=480 \mathrm{~m}$

Fig. 1. Sample of answer sheets, a sample problem and scoring in the experiment.

\section{Instruments}

\section{Materials}

A pre- and post-test were administered to all students before and after the experiment. Both of them were standardized tests using pencil-and-paper assessment which were identical to experimental tasks. All problems were about Newtonian mechanics and motion. They were based on word problems, which were expected to reflect students' capabilities in physics problem solving. The correlation between the pre- and post-test was 0.74 . Figure 1 is a sample of the answer sheet.

In order to necessitate cooperation during the experiment, we gave each student within each dyad five different hints which were formulated on the basis of Schoenfeld's five episodes of problem solving. The hints were randomly assigned to students. Figure 2 is the sample of hints given to different

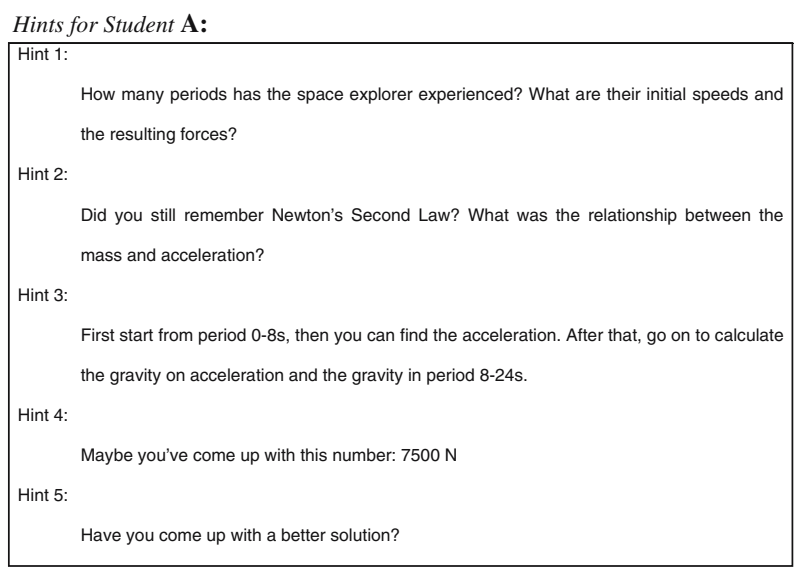

\section{Hints for Student $\mathbf{B}$ :}

Hint 1:
Reading the problem, you will notice that the explorer has experienced several periods. In
Hint 2:
each period, the initial speed and resulting force are different. Please list them.
constant?
Hint 3:
The acceleration in period $0-8 s$ is the acceleration from the resulting force while the
Hint 4:
acceleration in period $8-24 s$ comes from gravity.
Hint 5:

Have you come up with a better solution?

Fig. 2. Hints for students. 
students in one dyad. In pre- or post-tests there was no hint or any other kind of help for problem solving.

\section{Data Collection}

The data consists of students' pre- and post-test scores, and their written messages on the communication-log sheets. Students were asked to write down all the steps in the solution. Each step was scored according to its difficulty (see Figure 1). Students' pre- and post-test performances were used to verify our first research question, which was related to partner gender and learning achievement through cooperation. Students' communication-log sheets were used for analysis of communication style and of the content of the written messages in order to answer our second and third research questions. Written messages selected from Problems 1, 3 and 7 were analyzed with the modified Bales's IPA model for collecting information from students' interaction (Figure 3 shows the categories of analysis).

Based on Schoenfeld's five episodes, students' problem-solving processes during cooperation were

\begin{tabular}{ll}
\hline Units of interaction analysis & Examples: \\
\hline Ask for information & How many periods has the explorer experienced? \\
\hline Give Information & The explorer has experienced three periods: 0-8s, 8- \\
& 24s and 24-end. \\
\hline Ask for a suggestion & Should we write it down on the answer sheet? \\
\hline Make a suggestion & You'd better simplify your equations. \\
\hline Agree & Ithink you're right. \\
\hline Disagree & You shouldn't separate the man from the board. \\
\hline Tension & That's wrong. \\
\hline Uncertainty & \\
\hline
\end{tabular}

Fig. 3. Bales's interaction process analysis model (modified).

\begin{tabular}{|c|c|c|}
\hline $\begin{array}{l}\text { Schoenfeld's Five } \\
\text { Episodes } \\
\text { of Problem Solving }\end{array}$ & Definitions & Examples \\
\hline Reading the Problem & $\begin{array}{l}\text { understand the meaning of } \\
\text { the problem }\end{array}$ & $\begin{array}{l}\text { "Has the explorer experienced three } \\
\text { periods?" }\end{array}$ \\
\hline Exploring Knowledge & $\begin{array}{l}\text { relate information about the } \\
\text { problem to previously } \\
\text { learned knowledge }\end{array}$ & $\begin{array}{l}\text { "Which equation is related to mass, force } \\
\text { and acceleration?" } \\
\text { "Can we find the distance by using } \\
s=v_{o} t+1 / 2 a t^{2} \text { ?" }\end{array}$ \\
\hline $\begin{array}{l}\text { Analysis \& } \\
\text { Making a Plan }\end{array}$ & $\begin{array}{l}\text { break the information down } \\
\text { into several elements and } \\
\text { organize them }\end{array}$ & $\begin{array}{l}\text { "First start with 0-8s, and then find the } \\
\text { resulting acceleration. Next, look at 8-24s, } \\
\text { and then you can find gravitational } \\
\text { acceleration and gravity." }\end{array}$ \\
\hline Carrying out the Plan & $\begin{array}{l}\text { synthesize the information } \\
\text { gathered to develop an } \\
\text { answer }\end{array}$ & $\begin{array}{l}{ }^{" F} F=m a=1500^{*} 5=7500 \mathrm{~N} ; \\
G=m g=1500^{*} 10=15000 \mathrm{~N} a=10 \mathrm{~m} / \mathrm{s} 2 ; \\
\text { Solution: } F_{\text {result }}=F_{\text {push }}-G, \\
\rightarrow F_{\text {push }}=G+F_{\text {result }}=7500+3750 \mathrm{~N}=11250 \mathrm{~N} . "\end{array}$ \\
\hline Evaluation & $\begin{array}{l}\text { offer your own opinion } \\
\text { about the solution or idea }\end{array}$ & $\begin{array}{l}\text { "You can use a more direct way to solve } \\
\text { this problem by taking the man and the } \\
\text { board as a whole." }\end{array}$ \\
\hline
\end{tabular}

Fig. 4. Schoenfeld's five-episode problem solving.

analyzed using the written text on the communication-log sheets. The categories of problem-solving analysis are summarized in Figure 4.

Scoring of the statements in the communication$\log$ sheets was done with the help of these two systems and the scores were input into program MEPA (Erkens, 1998), which provides a database for input/ output and analysis of interaction. Each message written on the communication-log sheets was the basic unit of analysis.

\section{RESULTS}

\section{Learning Achievement}

We analyzed students' pre- and post-test performance in order to answer the first research question: the influence of partner gender on students' learning achievement. Previous research suggested that female students in single-gender cooperation would outperform females in mixed-gender cooperation, but that the same would not be the case for male students.

In an ANOVA test with "group" as independent factor and "pre-test" as dependent variable, we found that there was no significant difference among the four groups in pre-test scores $\left(F_{(3,46)}=1.50\right.$, $p>0.05)$. Neither was there a significant difference between females in $\mathrm{MG}$ and $\mathrm{FF}$ conditions 
Table I. Summary of Pre-test and Post-test Scores (Means and Standard Deviations) of Students in Four Groups

\begin{tabular}{|c|c|c|c|c|c|c|}
\hline \multirow[b]{2}{*}{ Conditions } & \multirow[b]{2}{*}{ Gender } & \multirow[b]{2}{*}{ Number of students } & \multicolumn{2}{|r|}{ Pre-test } & \multicolumn{2}{|c|}{ Post-test } \\
\hline & & & Mean & Standard deviation & Mean & Standard deviation \\
\hline \multirow[t]{2}{*}{ MG (12 dyads) } & Female & 12 & 76.58 & 12.27 & 77.17 & 9.65 \\
\hline & Male & 12 & 69.42 & 11.95 & $82.25^{*}$ & 9.57 \\
\hline FF (7 dyads) & Female & 14 & 65.79 & 15.20 & $79.14^{*}$ & 12.91 \\
\hline MM (6 dyads) & Male & 12 & 72.58 & 13.89 & 78.50 & 13.15 \\
\hline \multirow[t]{3}{*}{ Total } & Female & 26 & 70.77 & 14.71 & 78.23 & 11.34 \\
\hline & Male & 24 & 71.00 & 12.77 & 80.38 & 11.41 \\
\hline & Total & 50 & 70.88 & 13.68 & 79.26 & 11.31 \\
\hline
\end{tabular}

*Indicates a significant difference from females in the MG group $(p<0.01)$.

$\left(F_{(1,25)}=3.89, p>0.05\right)$, nor was there a significant difference between females and males in MG condition $\left(F_{(1,23)}=2.10, p>0.05\right)$. There was no interaction effect either. Table I shows the means and standard deviations of students' problem-solving performances for the pre- and post-tests.

The analysis of covariance (ANCOVA) of the students' post-test performance, using their pre-test scores as the covariate, showed that there was no significant difference between female and male students in learning performances. In general, females did as well as males on the post-test $\left(F_{(1,47)}=0.85\right.$, $p>0.05$ ). But there was a significant difference among the four groups $\left(F_{(3,42)}=4.87, p<0.05\right)$.

We conducted another ANCOVA with the four groups as the independent factor, the pre-test as the covariate and the post-test as the dependent variable. A pairs-wise comparison with Bonferroni adjustment was specified to examine the differences among the four groups. It turned out that females in the MG condition did significantly worse on the post-test than did females in the FF condition $\left(F_{(1,23)}=9.63, p<0.05\right)$ and males in the MG condition $\left(F_{(1,21)}=12.08, p<0.05\right)$. However, there was no significant difference on the post-test scores between males in $\mathrm{MG}$ and those in the MM conditions $\left(F_{(1,21)}=4.98, p>0.05\right)$.

So, females in the MG condition were at a significant disadvantage as compared with their male counterparts. Mixed-gender cooperation in physics favored male students over female students. However, when working in single-gender dyads, female students did just as well as male students. These results are in general agreement with other observations in the literature showing that mixed-gender cooperation disadvantages female students and that female students can learn more from a female than from a male partner. Compared with male students, female students' learning achievement is more sensitive to their partner gender. To explore why mixed-gender pairing had different influences on female and male students we analyzed the content and style of students' interaction during the problem-solving process.

\section{Interaction Style}

Messages about three problems were input into and analyzed in the MEPA program. Due to the presence of the teacher and the limited experiment time, there was very little room left for students to develop social talk that had no relation to the task. This was reflected in the high degree of on-task interaction among students. Of all 1113 written messages generated by students in the four groups, 1014 were identified as on-task interaction. Females in the MG condition had 229 on-task statements; females in the FF condition had 364; males in the MG condition had 285 and males in the MM condition had 136 on-task statements.

All the on-task messages were analyzed based on Bales's Interaction Process Analysis (Bales, 1950). The original twelve categories of interactive behavior units were condensed into nine to fit better into the problem-solving setting. Of the 1014 ontask statements, almost all (999) were able to be categorized. The total number of statements in the nine categories was counted. The results are summarized in Table II.

Most of the exchanged statements were from females in the FF condition (378), followed by males in the MG condition (256), females in the MG condition (224) and finally the males in the MM condition (141). In the MM condition there were not as many exchanges as in the other three groups.

To explore whether partner gender influenced students' interaction content, the chi-square test was 
Table II. Observed and Expected Number of Messages the Students Exchanged as Classified by nine Bales's IPA Systems

\begin{tabular}{|c|c|c|c|c|c|c|c|}
\hline \multirow{2}{*}{\multicolumn{2}{|c|}{ Bales's categories }} & & \multicolumn{4}{|c|}{ Groups } & \multirow[b]{2}{*}{ Total } \\
\hline & & & $\begin{array}{l}\text { Females in } \\
\text { mixed-gender } \\
\text { condition } \\
(N=12)\end{array}$ & $\begin{array}{c}\text { Females in } \\
\text { female-female } \\
\text { condition } \\
(N=14)\end{array}$ & $\begin{array}{c}\text { Males in } \\
\text { mixed-gender } \\
\text { condition } \\
(N=12)\end{array}$ & $\begin{array}{l}\text { Males in } \\
\text { male-male } \\
\text { condition } \\
(N=12)\end{array}$ & \\
\hline \multirow[t]{2}{*}{ A1 } & Ask for information & Observed number & 35 & 58 & 17 & 6 & $116^{*}$ \\
\hline & & Expected number & 26 & 44 & 30 & 16 & \\
\hline \multirow[t]{2}{*}{$\mathrm{A} 2$} & Give information & Observed number & 67 & 150 & 150 & 76 & $443^{*}$ \\
\hline & & Expected number & 99 & 167 & 114 & 63 & \\
\hline \multirow[t]{2}{*}{ B3 } & Ask for suggestion & Observed number & 45 & 48 & 21 & 6 & $120 *$ \\
\hline & & Expected number & 27 & 45 & 31 & 17 & \\
\hline \multirow[t]{2}{*}{ B4 } & Give suggestion & Observed number & 22 & 54 & 40 & 19 & $135^{*}$ \\
\hline & & Expected number & 30 & 51 & 35 & 19 & \\
\hline \multirow[t]{2}{*}{$\mathrm{C} 5$} & Disagree & Observed number & 5 & 6 & 3 & 2 & 16 \\
\hline & & Expected number & 4 & 6 & 4 & 2 & \\
\hline \multirow[t]{2}{*}{ C6 } & Agree & Observed number & 15 & 15 & 9 & 4 & $43 *$ \\
\hline & & Expected number & 10 & 16 & 11 & 6 & \\
\hline \multirow[t]{2}{*}{ D7 } & Tension & Observed number & 8 & 29 & 7 & 26 & $70^{*}$ \\
\hline & & Expected number & 16 & 27 & 18 & 10 & \\
\hline \multirow[t]{2}{*}{ D8 } & Rewards & Observed number & 9 & 6 & 5 & 0 & 20 \\
\hline & & Expected number & 5 & 8 & 5 & 3 & \\
\hline \multirow[t]{2}{*}{ E9 } & Uncertainty & Observed number & 18 & 12 & 4 & 2 & 36 \\
\hline & & Expected number & 8 & 14 & 9 & 5 & \\
\hline \multicolumn{2}{|c|}{ Total } & & 224 & 378 & 256 & 141 & 999 \\
\hline
\end{tabular}

conducted, using a significance level of 0.01 with four groups and the number of statements as factors. According to the test results, there was a statistically significant difference in the number of messages across the four groups $\chi^{2}(12)=72.47, p<0.001$.

To answer how partner gender influenced female students' and male students' interaction, the number of messages generated by students during problem solving was compared for each gender.

For female students chi-square tests showed significant differences between those in the FF condition and the MG condition in these categories: females in the MG condition were more likely to ask for suggestions and opinions $\chi^{2}(1)=2.85, p<0.1$, for example, as to when to submit the answer sheet or whether they should also draw the picture on the answer sheet. But they offered significantly less problem information $\chi^{2}(1)=4.47, p<0.05$, or suggestions about how to solve the problem $\chi^{2}(1)=11.16, p<0.05$. There was no significant difference between these two groups when looking at the other categories. The percentages of messages across all of the IPA categories are shown in Figure 5.

For male students more tension was found among those in the MM condition than in the MG condition $\chi^{2}(1)=26.99, p<0.05$. No significant

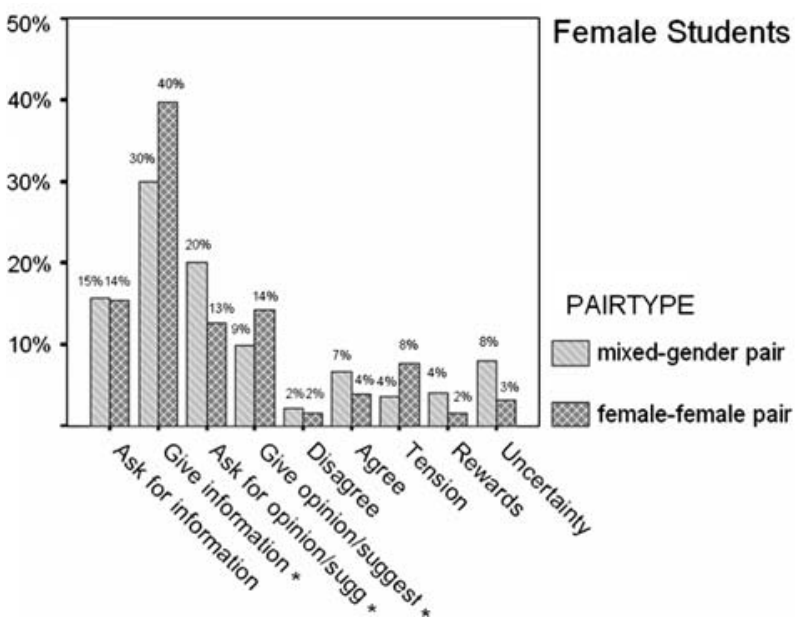

Fig. 5. Percentages of messages concerning the IPA model generated by female students in two conditions. *Significantly different from each other.

differences were found in the other categories. The percentages of messages across all of the IPA categories are shown in Figure 6.

Within the MG condition females' interaction differed greatly from that of males. Figure 7 shows the various message frequencies across all nine cate- 


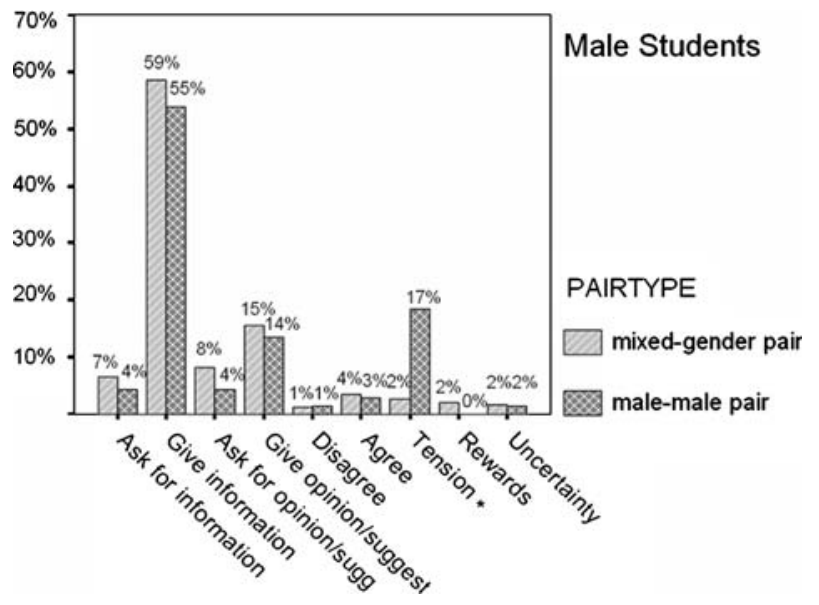

Fig. 6. Percentages of messages concerning the IPA model generated by male students in two conditions. *Significantly different from each other.

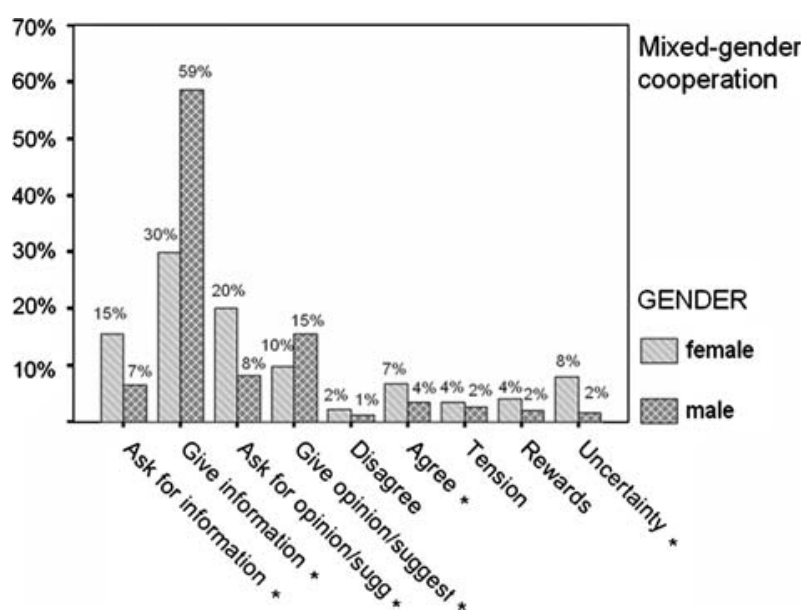

Fig. 7. Percentages of messages concerning the IPA model generated by female and male students in a mixed-gender condition. *Significantly different from each other.

gories. Compared with their male partners, female students asked significantly more often for problem information, $\chi^{2}(1)=11.26, p<0.05$, and for more suggestions about procedures, $\chi^{2}(1)=15.90$, $p<0.05$. Male students offered significantly more explanations about the problem, $\chi^{2}(1)=30.37$, $p<0.05$, and expressed their own opinions more often, $\chi^{2}(1)=2.72, p<0.1$. Females showed significantly greater uncertainty than did their male partners, $\chi^{2}(1)=12.35, p<0.05$. Females also generated more agreements than did their male partners, $\chi^{2}(1)=3.00, p<0.1$. No significant differences were found in other categories.
Qualitative study differences in interaction content were found mainly between the female and male students in the mixed-gender group. Females asked for information and suggestions more often than did males.

Below is an example of a communication protocol between Ping (female) and $\mathrm{Li}$ (male) in a mixedgender condition. Here Problem 1 (shown in Figure 1) was used, and this example mirrors the differing communication styles of female and male students.

Li (male): The explorer experienced three periods.

Ping (female): What was the gravity?

Li (male): Look!! $a_{(0-8 \mathrm{~s})}=40 / 8=5 \mathrm{~m} / \mathrm{s}^{2}, a_{(8-24 \mathrm{~s})}=$ gravity $\rightarrow a=40 /(24-8)=2.5 \mathrm{~m} / \mathrm{s}_{2}$. Is that right? I'm not sure.

$\mathrm{Li}$ (male): Well, gravity $=m g=1,500 \times 2.5=3,750$, but check it! Maybe I'm wrong.

Ping (female): I don't understand.

$\mathrm{Li}$ (male): The explorer is on a star.

Ping (female): So?

Li (male): Well, gravity would be different from Earth's.

Ping (female): Yes, but it's still not very clear.

Li (male): Okay, within this period $0-8 \mathrm{~s}, F_{\text {motor }}-m g=m a$. See?

Ping (female): And...

Li (male): The motor has got a problem. So during 8-24 s, it's in free-fall, and here $a$ is the acceleration due to gravity.

Ping (female): Okay, I think I get it now. You'd better write the process down on the answer sheet.

(Li filled in the answer sheet while Ping was watching.)

Ping (female): Should we ask for a worked-out example?

Examining the dialogue between Ping and $\mathrm{Li}$, we found that most of the specific suggestions or information was given by $\mathrm{Li}$, while Ping's responses showed a higher level of uncertainty. From the beginning she had difficulty in understanding gravity, but she only asked about it once. Even after Li's explanation she still didn't grasp it. But she didn't raise the same question again. Instead she used phrases like "not very clear" as a hint to Li to explain it in more detail.

An example of communication protocols between Nan and Fang was typical for females in the FF condition.

Nan: The explorer has experienced several periods.

Fang: Three periods: accelerating, then decelerating and accelerating again.

Nan: I think so, too, so the direction of "a" should be: accelerating upwards, then decelerating upwards and finally accelerating downwards.

Fang: Okay. But within the period of $8-24 \mathrm{~s}$, is gravity acceleration $\rightarrow a=g$ ?

Nan: What is $g$ ? We're not on Earth.

Fang: "Not on Earth" means what? Should it be $1 / 6 G$ ?

Nan: No. $g=9.8 \mathrm{~m} / \mathrm{s}^{2}$ only can be used for things on Earth.

Fang: So $g$ should be calculated in this problem, right? 
Nan: Right. But first we should start with the period 0-8 s. $v_{\mathrm{t}}=40 \mathrm{~m} / \mathrm{s} v_{0}=0 \mathrm{~m} / \mathrm{s} t=8 \mathrm{~s} \rightarrow a=\left(v_{\mathrm{t}}-v_{0}\right) / t=40 /$ $8=5 \mathrm{~m} / \mathrm{s}^{2}$.

Fang: I see. $v_{0}=40 \mathrm{~m} / \mathrm{s} v_{\mathrm{t}}=0 \mathrm{~m} / \mathrm{s} t=(24-8)=16 \mathrm{~s} \rightarrow$ $g=\left(v_{\mathrm{t}}-v_{0}\right) / t=40 / 16=2.5 \mathrm{~m} / \mathrm{s}^{2}$

Nan: Right

Nan: Okay, write it down on the answer sheet.

Like Ping, Fang in this case also had some problem in understanding $g$ at the outset. But she argued with her partner, Nan, and freely posited her understanding by guessing whether $g$ equaled $1 / 6 G$. She further deduced from Nan's explanation that $g$ should be calculated and tried it once. Unlike Ping she didn't use ambiguous words to show her uncertainty, but asked questions and posited her own conceptions more directly.

The dialogue below between Tao and Wang is typical for interactions in the MM condition.

Tao: $h=20 \times 8+20 \times 16=20 \times 24=480 \mathrm{~m}$

Tao: $v_{\mathrm{t}}=v_{\mathrm{o}}+a_{\mathrm{t}}, 8 a=v_{\mathrm{t}}-v_{0}=40, a=5 \mathrm{~m} / \mathrm{s}^{2}$

Wang: Wrong. During $8-24 \mathrm{~s}$, it has already started falling.

Tao: No, it's just flying upwards with decreasing speed.

Wang: At the 24th second, $v=0$, then starts free-fall.

Tao: And then $v$ changes from increasing to decreasing. When $v=0$, it reaches its highest point.

Wang: Oh that's right!

Tao: $F=m a=1500 \times 5=7500 \mathrm{~N}$.

Tao: $F_{\text {pull }}=15000+7500=22500 \mathrm{~N}, H_{\max }=480 \mathrm{~m}$.

Neither Wang nor Tao asked their partner whether their solution was right. Using the words "wrong" or "no," they argued over their partner's work directly instead of admitting that they didn't understand. In contrast, $\mathrm{Li}$ in the MG condition showed Ping twice that he was not sure of the solution. It could be tentatively concluded then that partner gender significantly influenced the female students' interaction, and only slightly influenced the male students' interaction. The interaction of female-female dyads was much better balanced than was that of the mixed-gender dyads. Males in mixed-gender dyads showed giving-and-explaining behavior more than did their female partners. The differences in verbal interaction have provided a basis for further analysis of students' cognitive elaboration during cooperative problem solving.

\section{Problem-Solving Episodes}

The third purpose of the study was to find out whether there was an influence from partner gender on students' problem-solving activities during cooperation. Students' written messages were coded according to Schoenfeld's five episodes of problem solving (Figure 4). The observed and expected numbers of statements are presented in Table III.

Of the 1014 on-task messages, $814(80 \%)$ were identified as students' problem-solving activities. There is a significant difference among the four groups of students as to the numbers of statements about the five episodes $\chi^{2}(12)=72.47, p<0.01$. On average males in the MM condition and females in the MG condition made fewer statements concerning problem solving than did females in the FF condition or males in the MG condition. Females in the FF condition exchanged the most statements on problem solving as compared with the other three groups. It is then worth investigating during which episode of

Table III. Observed and Expected Number of Statements Concerning Problem-Solving Episodes in Four Groups of Students

\begin{tabular}{|c|c|c|c|c|c|c|}
\hline & & \multicolumn{4}{|c|}{ Groups } & \multirow[b]{2}{*}{ Total } \\
\hline & & $\begin{array}{l}\text { Female in } \\
\text { mixed-gender } \\
\text { condition }\end{array}$ & $\begin{array}{c}\text { Female in } \\
\text { female-female } \\
\text { condition }\end{array}$ & $\begin{array}{c}\text { Male in } \\
\text { mixed-gender } \\
\text { condition }\end{array}$ & $\begin{array}{l}\text { Male in } \\
\text { male-male } \\
\text { condition }\end{array}$ & \\
\hline \multirow[t]{2}{*}{ Read problem } & Observed number & 45 & 42 & 40 & 19 & \multirow[t]{2}{*}{$146^{*}$} \\
\hline & Expected number & 42 & 21 & 23 & 24 & \\
\hline \multirow[t]{2}{*}{ Explore knowledge } & Observed Number & 43 & 63 & 50 & 36 & \multirow[t]{2}{*}{$192 *$} \\
\hline & Expected number & 53 & 41 & 39 & 60 & \\
\hline \multirow[t]{2}{*}{ Make a plan } & Observed number & 28 & 100 & 100 & 53 & \multirow[t]{2}{*}{$281^{*}$} \\
\hline & Expected number & 50 & 96 & 113 & 128 & \\
\hline \multirow[t]{2}{*}{ Implement the plan } & Observed number & 4 & 24 & 27 & 1 & \multirow[t]{2}{*}{56} \\
\hline & Expected number & 1 & 5 & 6 & 1 & \\
\hline \multirow[t]{2}{*}{ Reflection } & Observed number & 37 & 63 & 32 & 7 & \multirow[t]{2}{*}{$69^{*}$} \\
\hline & Expected number & 33 & 30 & 18 & 0 & \\
\hline Total & & 157 & 292 & 249 & 116 & 814 \\
\hline
\end{tabular}


problem solving students exchanged relatively more information.

For females students, as illustrated in Figure 8, those in the MG condition generated significantly more statements paraphrasing the problem information than did those in the FF condition $\chi^{2}(1)=5.44$, $p<0.05$, while the latter generated more statements about the solution plan $\chi^{2}(1)=13.88, p<0.05$, and did significantly more on calculations $\chi^{2}(1)=4.02$, $p<0.05$. No significant differences were found in other episodes.

Figure 9 shows the percentages of messages across the five problem-solving episodes for male students. Males in MG conditions did significantly

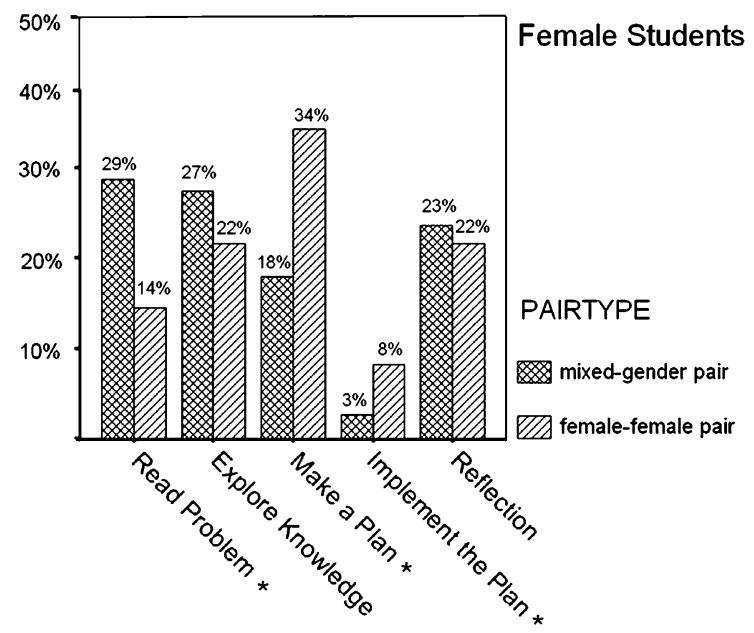

Fig. 8. Percentages of messages concerning Schoenfeld's problemsolving episodes generated by female students in two conditions. *Significantly different from each other.

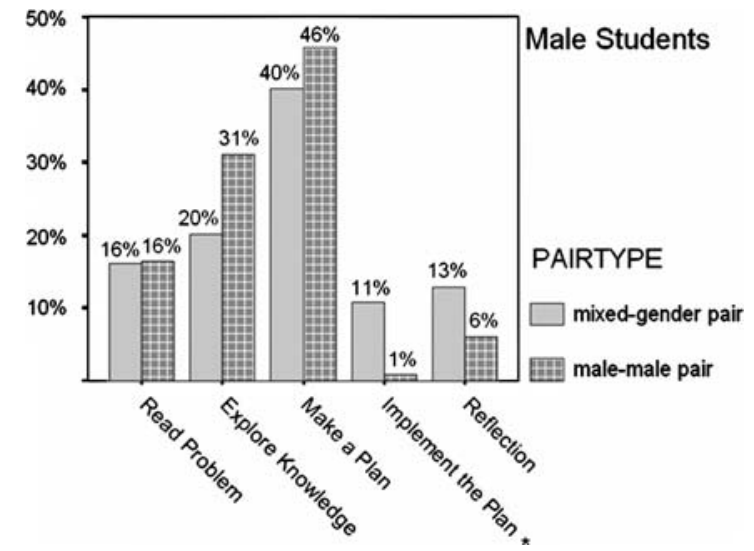

Fig. 9. Percentages of messages concerning Schoenfeld's problemsolving episodes generated by male students in two conditions. *Significantly different from each other. more calculations than did those in MM conditions $\chi^{2}(1)=14.11, p<0.05$. For the other episodes chi-square tests found no significant differences (Figure 9).

Figure 10 shows the percentages of messages between male and female students within MG conditions across the five episodes. Male students generated significantly more statements about mapping a solution plan $\chi^{2}(1)=37.09, p<0.05$, and did more calculations $\chi^{2}(1)=13.92, p<0.05$ than did their female partners. There were no significant differences between these two groups in other episodes.

Qualitative study of students' protocols showed the differences in the problem-solving process among the four groups. The example of Li (male) and Ping (female) given above indicated that mixed-gender cooperation hindered female students from going further in problem solving. Ping only asked once what the $g$ was. After Li explained it three times, Ping said, "I think I know now." And then she urged Li to write down the answer. One might doubt whether Ping really did understand Li's explanation of this problem. The protocol showed that Li actually didn't give any explicit explanation. But Ping ended the dialogue by saying then that she now knew. Doing so kept her from being a skilled problem solver.

In contrast, females in the FF condition had relatively more statements on the last three episodes of problem solving. Unlike Ping, Fang tried to calculate the $g$ by herself. She applied the knowledge actively and synthesized the equations in order to develop her own answer. She was involved in the application and internalization of the knowledge more than Ping was.

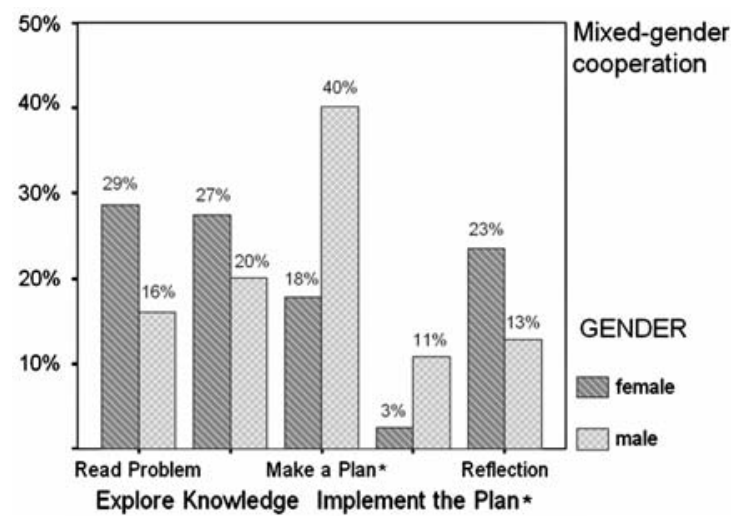

Fig. 10. Percentages of messages concerning Schoenfeld's problem-solving episodes generated by female and male students in a mixed-gender condition. *Significantly different from each other. 
For male students, as shown in Figure 10, those in the MM condition were more concerned with recalling prior knowledge while those in the MG condition were more concerned with working out the plan. In the MG condition, $11 \%$ of the statements generated by males concerned calculating and working out the plan in contrast to only $3 \%$ among males in the MM condition. This was also reflected in the example of Ping and Li. In mixed-gender cooperation, we found that males valued their female partner's opinions, and female partner's questions propelled them to deliberate on the problem information.

\section{CONCLUSIONS}

The questions addressed in this research concerned whether partner gender influenced students' learning achievement in physics as far as their communication and problem-solving activities in cooperative problem solving were concerned, and whether female students' learning was more sensitive to partner gender than was that of male students. Specifically, our purpose was to unravel gender effects in cooperative learning through the lens of interaction styles and the problem-solving process. Both quantitative and qualitative methods were used to analyze students' problem-solving performance and interaction content. We studied the cognitive activities of students in four gender groups: females in the MG condition, females in the FF condition, males in the MG condition and males in the MM condition. The students in all four groups worked in dyads.

For female students, analyses of pre- and posttest performances showed that partner gender was a significant factor in their learning achievement. Females in female-female dyads significantly outperformed females in mixed-gender dyads. Within mixed-gender dyads, males learned much better than females. These results are congruent with previous research that found females were at a disadvantage in mixed-gender cooperation (Barbieri and Light, 1992; Light et al., 2000). They did better in single-gender peer learning.

To explore the reason that mixed-gender cooperation disadvantaged female students, we examined students' communication protocols and analyzed their written messages according to Bales's IPA model and Schoenfeld's five episodes of the problem-solving process. Our analyses of students' interactions indicated that females in mixed-gender dyads asked for information or suggestions more often, while their male partners were much more likely to provide help and offer suggestions. This indicated, to some degree, that females had less confidence in their knowledge of physics or in their problem-solving ability than did their male counterparts. A great degree of uncertainty from females can be detected during mixed-gender cooperation. Analyses of their problem-solving processes showed that males assumed the task of planning and calculating the problem, while their female partners put more effort into paraphasing the problem information. It appeared that female students had difficulty in arguing or positing their own understanding while working with a male partner. During problem solving females were more likely to focus on the literal meaning of the problem rather than systematically analyzing it.

Unlike females in mixed-gender dyads, females in female-female dyads were not submissive and not less confident in their abilities. They discussed problems by giving information and made their own suggestions. They didn't ask as many questions, as did those in mixed-gender dyads. Analysis of their problem-solving process showed that females in female-female dyads generated the most messages concerning planning and calculation among the four groups. They put forward their ideas freely and were actively more involved in problem solving. Cooperation between females was better balanced than was that of mixed-gender dyads.

Cooperation between males was not as harmonious as that between male and female students. Males complained more while working with a male partner, for example, about how complicated the problems were and why they should stay in the physics classroom. Another interesting thing to note was that males in mixed-gender dyads did a lot of calculating tasks while males in male-male dyads did very little calculating. After examining their answer sheets, we found that most males in male-male dyads carried the working plan out directly in the answer sections rather than on the communication-log sheets. Once they had come up with some idea and agreed with each other on it, they moved to the answer sheets, calculating and revising there. But males in mixed-gender dyads first wrote down all the steps of problem solving on the communication-log files, making sure that their female partners agreed, and then copied them onto the answer sheets. But, generally speaking, their learning achievement and problem-solving activities were not significantly influenced by partner gender. 
Our results point out that during cooperation partner gender is not only a significant factor for females' learning achievement but also for their interaction and problem-solving activities. Compared with male students, female students' interaction and problem-solving activities were more sensitive to their partner's gender in cooperative learning, and this might explain why their learning achievement was significantly influenced by their partner's gender.

\section{SUGGESTIONS AND LIMITATIONS}

This is a preliminary study for research whose goal is to gain an insight into gender difference in the CSCL (Computer-Supported Collaborative Learning) environment. Previous research has tended to study female and male behaviors directly in a computer-involved setting. However, the computer itself is arguably a disadvantage for females. Since female and male students have different types of exposure to computer technology (Fetler, 1985), results of previous empirical studies cannot be expected to explain female students' real interaction and problem-solving activities during cooperative problem solving. In light of this, there is a need to study students' communication and cognitive thinking without being "threatened" by computers.

The study has shed light onto the "blackbox" of cooperation by going into a detailed analysis of students' interaction and working processes. Still, there are some limitations to this study. One is the small size of our samples. This might explain why we were unable to explore whether there was a significant effect from self-confidence or emotions on male and female students in mixed-gender dyads. Second, we have not explored the effect of students' preferences, such as whether they preferred working in a mixed or a single-gender dyad. Another limitation was the scope of the program in this study. It is necessary to provide a longer series of lessons for problem-solving learning (Pol et al., 2005) in order to let students get used to solving problems through cooperation. In the space of eight lessons it was hard to assess students' development of problem-solving skills. A longer study would be recommended for future research. Finally, it remains an open question whether the results in this study in China could also be applicable to countries with a different cultural background.

For future study the outcome of this study should be verified through more studies with the same design. There should be firm empirical evidence drawn from different cultures. Since the ultimate goal of education is to prepare students to work effectively in various social conditions, which are not simply those limited to single-gender groups (Speck, 2003), we also suggest investigating further why females do not perform well in cooperative problem solving with males and how this can be improved upon. One could try to discover whether there is a causal relationship between the communication style and content of both female and male students in mixed gender groups and whether this is a factor in their differences in learning gains. Our hypothesis is that females in mixed dyads are reluctant to put forward their ideas because they do not feel very self-confident. Females first tend to ask for information and suggestions and do not respond directly to their male partner's inquiries. Due to this, males start putting forward their own ideas, rather than waiting for the females to understand their solutions fully.

An interesting approach would be to boost female students' self-confidence when working with male students. For instance, we suggest a specially designed computer program for cooperative problem solving that provides "hints" as just-in-time instruction for female students. To avoid making female students feel that they are less capable in physics, we suggest that the use of hints should not be compulsory. Female students should make their own decisions whether they need hints for help. It could facilitate female students' cognitive elaboration and enhance their self-confidence while working with male partners (Ding and $\mathrm{Xu}$, 2005). The computer program also would make it possible to check the suggestions their partner makes. Perhaps that could smooth out the gender difference in communication by giving females information to put forward during cooperation.

\section{REFERENCES}

Bales, R. F. (1950). Interaction Process Analysis: A Method for the Study of Small Groups, Addison-Wesley, Reading, MA.

Bales, R. F. (1999). Social Interaction Systems: Theory and Measurement, Transaction, New Brunswick.

Barbieri, M. S., and Light, P. H. (1992). Interaction, gender, and performance on a computer-based problem solving task. Learning and Instruction 2: 199-213.

Cohen, E. (1994). Restructuring the classroom: Situations for productive small groups. Review of Educational Research 64(1): 1-35.

Ding N., Xu Y. R. (2005) “Giving students hints"-An investigation of improving students' problem-solving skills in high school science learning. Asia-Pacific Forum on Science Learning 6(2).

Erkens, G. (1998). Multiple Episode Protocol Analysis (MEPA 3.0), Internal publication. Department of Educational Sciences, Utrecht University, The Netherlands.

Fetler, M. (1985). Sex differences on the California statewide assessment of computer literacy. Sex Roles 13(3/4): 181-191. 
Hogan, D., and Tudge, J. (1999). Implications of Vygotsky's theory for peer learning. In A. O'Donnell, \& A. King (Eds.), Cognitive Perspectives on Peer Learning Mahwah, NJ: Lawrence Erlbaum Associates.

Howe, C., Tolmie, A., Greer, K., and Mackenzie, M. (1995). Peer collaboration and conceptual growth in physics: Task influences on children's understanding of heating and cooling. Cognition and Instruction 13(4): 483-503.

Hyde, J. S., Fennema, E., and Lamon., S. J. (1990). Gender differences in mathematics performance: A meta-analysis. Psychological Bulletin 107(2): 139-155.

Johnson, D. W., and Johnson, R. T. (1986). Computer-assisted cooperative learning. Educational Technology 26(1): 12-18.

Lakoff, R. (1973). Language and woman's place. Language in Society 2: 45-80.

Lay, M. M. (1992). The androgynous collaborator: The impact of gender studies on collaboration. In J. Forman (Ed.), New Visions of Collaborative Writing (pp. 82-104). Portsmouth, NH: Boynton/Cook.

Lehtinen, E. (2003). Computer-supported collaborative learning: An approach to powerful learning environments. In Powerful Learning Environments: Unravelling Basic Components and Dimensions, Pergamon, pp. 35-53.

Li, Q. (2002). Gender and computer-mediated communication: An exploration of elementary students' mathematics and science learning. Journal of computers in mathematics and science teaching 21(4): 341-359.

Light, P., Littleton, K., Bale, S., Joiner, R., and Messer, D. (2000). Gender and social comparison effects in computer-based problem solving. Learning and Instruction 10: 483-496.

Margrett, J. A., and Marsiske, M. (2002). Gender differences in older adults' everyday cognitive collaboration. International Journal of Behavioral Development. 26(1): 45-59.

Mercer, N. (1996). the quality of talk in children's collaborative activity in the classroom. Learning and Instruction 6: 359-377.

Orenstein, P. (1994). School Females, Doubleday, NY.
Pol, H., Harskamp, E., and Suhre, C. (2005). The solving of physics problems: Computer assisted instruction. International Journal of Science Education 27: 451-469.

Schoenfeld, A. H. (1992). Learning to think mathematically: Problem solving, metacognition, and sense making in mathematics. In D. A. Grouws (Ed.), Handbook of Research on Mathematics Teaching and Learning (pp. 334-367). NY: Macmillan.

Schwartz, D. L. (1995). The emergence of abstract representations in dyad problem solving. Journal of the Learning Sciences 4(3): 321-354.

Sharan, S., and Shachar, H. (1988). Language and learning in the cooperative classroom, Springer, New York.

Sherin, B. L. (2001). How students understand physics equations. Cognition and Instruction 19(4): 479-541.

Siann, G., and Macleod, H. (1986). Computers and children of primary school age: issues and questions. British Journal of Educational Technology 17: 199-44.

Siann, G., Durndell, A., Macleod, H., and Glissov, P. (1988). Stereotyping in relation to gender gap in participation in computing. Educational Research 30: 98-103.

Speck, B. W. (2003). Fostering collaboration among students in problem-based learning. In New Directions for Teaching and Learning, Wiley Periodicals, Inc. p. 59, 95.

Sutherland, L. (2002). Developing problem solving expertise: The impact of instruction in a question analysis strategy. Learning and Instruction 12: 155-187.

Teasley, S. (1995). The role of talk in children's peer collaboration. Developmental Psychology 3(2): 207-220.

Underwood, G., Jindal, N., and Underwood, J. D. M. (1994). Gender differences and effects of co-operation in a computerbased language task. Educational Research 36: 63-74.

Webb, N. M. (1984). Sex Differences in Interaction and Achievement in Cooperative Small Groups. Journal of Educational Psychology 36(1): 33-44. 